\title{
Modelling and Simulation for Major Incidents
}

\author{
An Innovative Approach to Medical Response
}

\author{
Eleonora Pacciani ${ }^{1}$, Alessandro Borri ${ }^{2}$, Paolo Maurizio Soave ${ }^{3}$, Daniele Gui $^{3}$, Sabina Magalini ${ }^{3}$, \\ Simona Panunzi ${ }^{2}$, Claudio Roberto $\mathrm{Gaz}^{2}$, Pasquale Gaudio ${ }^{1}$, Andrea Malizia ${ }^{1}$, Andrea De Gaetano ${ }^{2}$ \\ ${ }^{1}$ University of Tor Vergata, Rome, Italy \\ e-mail: eleonora.pacciani@virgilio.it,\{gaudio,malizia\}@ing.uniroma2.it \\ ${ }^{2}$ CNR-IASI Biomathematics Laboratory, Rome, Italy \\ e-mail:\{alessandro.borri,simona.panunzi,claudior.gaz,andrea.degaetano\}@biomatematica.it \\ ${ }^{3}$ Catholic University of the Sacred Heart, Rome, Italy \\ e-mail: pmsoave@yahoo.it,\{daniele.gui,sabina.magalini\}@rm.unicatt.it
}

\begin{abstract}
In recent years, there has been a rise in Major Incidents with big impact on the citizens health and the society. Without the possibility of conducting live experiments when it comes to physical and/or toxic trauma, only an accurate in silico reconstruction allows us to identify organizational solutions with the best possible chance of success, in correlation with the limitations on available resources (e.g. medical team, first responders, treatments, transports, and hospitals availability) and with the variability of the characteristic of event (e.g. type of incident, severity of the event and type of lesions).

Utilizing modelling and simulation techniques, a simplified mathematical model of physiological evolution for patients involved in physical and toxic trauma incident scenarios has been developed and implemented. The model formalizes the dynamics, operating standards and practices of medical response and the main emergency service in the chain of emergency management during a Major Incident.
\end{abstract}

Keywords - predictive models for adverse outcomes; personalized patient treatment; health monitoring applications

It is today clear that the incidence of Major Incidents (MIs) - situations where available resources are insufficient for the immediate need of medical care - has increased significantly parallel to the technical and economical development in the world [1]. The World Disaster Report 2007 showed a 60\% increase in the occurrence of incidents defined as major during the decade 1997-2006 [2]. During the last decade, the reported deaths from such incidents increased from 600,000 to more than 1,200,000 and the number of affected people increased from 230 to 270 million [2]. MIs have previously been considered as low probability events that might inflict bodily harm, incapacitation, or even fatalities, and however have a big impact on the citizens and the society [4].

The main causes of this increase have been recognized in [5]: the improvement in global population which means a risk factor in itself; the escalation of natural disasters; the tons of flammable, explosive, chemical, and toxic agents which are produced, transported on roads and railroads, and used every year; the global terrorism (e.g., chemical warfare agents (CWA), biological warfare agents (BWA), and radiological and nuclear particulate hazards); the continuing urbanization, which means increasing number of people in crowded areas for living or gathering together for public events. Such areas are also potential targets for terroristic attacks or a risk in themselves, because many people are collected in limited areas, which can be difficult and time-consuming to evacuate (e.g., in case of structural collapse or fire).

The price we have to pay is the risks connected to this development. Parallel to this, and as a paradox, the vulnerability of our health care system to such situations has increased: increasing demands on efficiency reduce or eliminate the "resilience capacity" for high loads of casualties [1].

The goal of the health care system during MI is to reduce or eliminate loss of life and health, and subsequent physical and psychological suffering [6].

The achievement of such a goal requires two actions [6]:

1) Relocating available resources to where they are most needed and rapid mobilization of additional resources (personnel and materials);

2) Optimal utilization of available resources through accurate priorities between patients and between measures and through the use of simplified methods for triage, treatment and transport [7].

Relocation and mobilization of resources can be enhanced by the introduction and proliferation of good and accurate mathematical models to manage incident medical response [8], for example to simulate physiological value, predict adverse outcomes and personalize the treatment of the patients. 
Advanced simulation models can illustrate all components in the chain of MI management (e.g., patient evolution, triage, treatment, transport, and hospital) and aim to developing and ensuring resilience capacity. The improvement of resilience and the better integration of health care systems in real operations will enhance the safety and security of citizens.

\section{METHODS}

Utilizing modelling and simulation techniques, we have developed and implemented a mathematical model for the physiological patient evolution during/after physical and toxic trauma.

\section{A. Taxonomy}

For the implementation of a mathematical model of physiological patient evolution during a MI, it has been used the following taxonomy, which involves the following classes:

- Events: an event is an accident or an incident that involves a certain amount of people. We have built an Event Library, which contains physical and toxic trauma incidents [3,9-11]:

- motorway accident;

- bridge collapse;

- ship explosion;

- train crash.

- stadium crush;

- accident/incident with Irritants with High Water Solubility (IHS);

- accident/incident with Irritants with Low Water Solubility (ILS);

- military accident/incident with Yperite (Mustard Gas, Y);

- terroristic attack with Nerve Agents (NA).

- Lesions: a lesion is a damage or an injury that can afflict in general all the systems of the human organism. An event is liaised to a set of lesions with a conditional probability of occurrence. A Lesions Library has been built, containing physical and toxic lesions [3,12,13-15,9-11,16]:

- bleeding wound;

- internal bleeding;

- burn (i.e., skin, and airway mucosa);

- hypothermia;

- head trauma;

- facial trauma;

- chest trauma;

- spinal trauma;

- abdominal trauma;

- pelvic trauma;

- extremity trauma;

- myocardial ischemia;

- myocardial infarction;

- irritation (i.e., mucous membrane, and conjunctiva);

- desquamation (i.e., skin, and airway mucosa);
- ulceration (i.e., skin, corneal, and airway mucosa);

- necrosis (i.e., liquefactive (e.g., due ammonia), coagulative (tissue), and organ failure);

- edema (i.e., upper airway (e.g., faring, larynx), noncardiogenic pulmonary, and conjunctiva);

- atelectasis;

- miosis.

- Physiology: in agreement with the ABCDE Primary Survey and Resuscitation [3], there are only five main ways to die, from fatal complication involving:

- $\operatorname{Airway}(\mathrm{A})$;

- Breathing (B);

- Circulation $(\mathrm{C})$;

- Disability Nervous System (D);

- Extra Damage or Exposure with Environmental Control (E).

Accordingly, the patient dynamics can be described by a set of physiological variables, based on $\mathrm{ABCDE}$ paradigm. The set of physiological variables consists of 10 variables:

- A1 (i.e., intact, at risk, partially obstructed, or completely obstructed airway);

- B1 (i.e., respiratory rate);

- B2 (i.e., tidal volume);

- B3 (i.e., oxygen saturation, $\mathrm{SpO} 2$ );

- C1 (i.e., heart rate);

- $\quad$ C2 (i.e., Mean Arterial Pressure, MAP);

- D1 (i.e., Glasgow Coma Scale, GCS);

- D2 (i.e., seizures);

- D3 (i.e., cholinergic activity);

- $\quad$ E1 (i.e., trauma, burns, and contamination).

- State Variables: the patient dynamics is described by:

- $x(t)$, which is the current state of each variable;

- $v(t)(i . e . d x(t) / d t)$, which is the speed at which each variable changes its state.

- Therapeutic maneuvers: there is a set of therapies (according to the ABCDE treatment) repairing the damage that afflicts the physiological variables. We have built a Therapeutic Maneuvers Library, which contains [12,17-20]:

- decontamination;

- oxygen;

- intubation;

- ambu-bag;

- Hyperbaric Oxygen Therapy (HBOT);

- saline;

- blood;

- vascular surgery;

- neural surgery;

- orthopedic surgery; 
- tourniquet;

- respiratory drugs (e.g., bronchodilators, respiratory stimulants);

- cardio drugs (e.g., $\beta$-adrenergic agonist as adrenaline, chronotropes as atropine);

- neuro drugs (e.g., anticonvulsant drugs as benzodiazepines).

- Lesions/Maneuvers delta-alpha matrices: each lesion affects one or more physiological variables with a maximal initial damage (delta ${ }^{-}$) and a maximal worsening rate per unit time (alpha'); symmetrically, each therapeutic maneuver repairs one or more physiological variables with a maximal initial improvement $\left(\right.$ delta $\left.^{+}\right)$and a maximal improvement rate per unit time (alpha ${ }^{+}$).

- Assets: an asset is characterized by a collection of therapeutic maneuvers. An Assets Library has been built, which contains:

- ambulance;

- emergency room;

- decontamination team;

- operating theatre;

- police car;

- $\quad$ on the scene (i.e., the absence of therapies).

The assets link the patient model to a future logistic model, which takes into account the real-time availability of the resources.

\section{B. Mathematical Model}

The mathematical model describes the physiological patient evolution in terms of piecewise-linear trajectories in the state space, where the patient dynamics is described by means of normalized physiological values (see previous section for more details). In the normal form of first order, the evolution of each variable satisfies the following differential equation:

$$
\frac{d x(t)}{d t}=-\alpha+u(t) \quad t \geq t_{0}
$$

starting from the initial condition:

$$
x\left(t_{0}\right)=1-\Delta
$$

where:

- $t_{0}$ is the start of the event;

- $\quad \mathrm{x}(\mathrm{t})$ is the value assumed by each physiological variable at time $t \geq t_{0}$, when the damage of each variable starts. Each variable takes values in $[0,1]$, where 1 is the initial healthy value, and has a lower-bound value under which the patient's health is compromised;

- $\quad \mathrm{x}\left(\mathrm{t}_{0}\right)$ is the value assumed by each physiological variable at time $\mathrm{t}=\mathrm{t}_{0}$;

- $\quad \mathrm{dx}(\mathrm{t}) / \mathrm{dt}=\mathrm{v}(\mathrm{t})$ is the speed at which each variable changes its state;

- $\Delta: \Delta \epsilon[0,1]$ is the maximal initial damage at time $\mathrm{t}_{0}$
- $\alpha: \alpha \in \mathbb{R}_{0}^{+}$is the maximal worsening rate [relative damage/unit time];

- $\quad \mathrm{u}(\mathrm{t})$ is a non-negative therapy component.

However, the event starts affecting the patient's status at (possibly) different times for each individual, causing lesions, namely reductions in the value of one or more physiological variables.

\section{RESULTS}

To run simulations, the following functions have been implemented in Matlab:

- GeneratePatients: this function randomly generates patients, affected by different lesions. The degree of severity of each patient can be sampled according to different (choosable) distributions: gaussian, uniform or triangular;

- EvolvePatients: this function simulates the patients evolution from (1)-(2), with and without therapeutic maneuvers;

- TimeToDeath: this function calculates the time to death for each patient, if there is not a medical treatment with therapeutic maneuvers;

- TriagePatients: this function simulates a patients triage, based on the time to death, and gives the color code according to literature review [21,22].

\section{A. Simulation 1}

Simulation 1 shows results from three different event severities taking values in $[0,1]$, where 1 is the maximum level:

1.a Maximum event severity $=1$;

1.b Medium event severity $=0.6702$;

1.c Low event severity $=0.3822$.

Common features of scenario:

- $\quad$ event code = IHS;

- $\quad$ number of people in area $=1000$;

- $\quad$ event radius $=100[\mathrm{~m}]$;

- $\quad$ severity probability distribution = gaussian;

- $\quad$ time horizon $\mathrm{T}=100[\mathrm{~h}]$.

High-severity events affects a much larger number of people than low-severity events (Fig. 1). An unintuitive effect of this is that the relative percentage of red codes may decrease (Fig. 3).

Note that the number of patients alone (Fig. 1) does not determine the severity of event and the declaration of MI for the Health Services. Small incidents with relatively few casualties (Fig. 1) can therefore require early involvement of regional or national resources. Following the classification scheme for MIs in [23], based on the resources required for a response, this type of incidents (i.e. small) could be assessed as a Level III incident (where state or federal aid is needed). The classification system (maximum, medium, and low severity) shows that the resources required for the medical response are 
not always directly proportional to the severity of the event (Fig. 2-3).

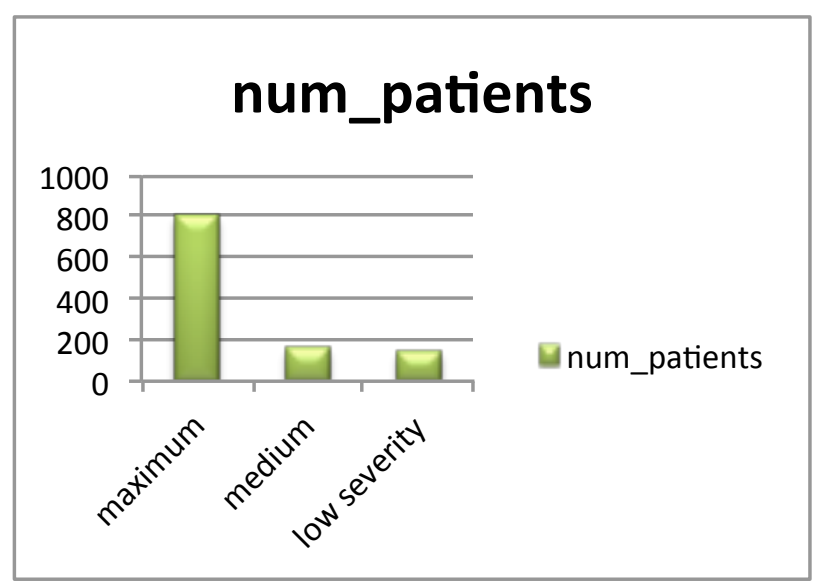

Fig. 1. Number of patients for each event severity level (maximum, medium, low).

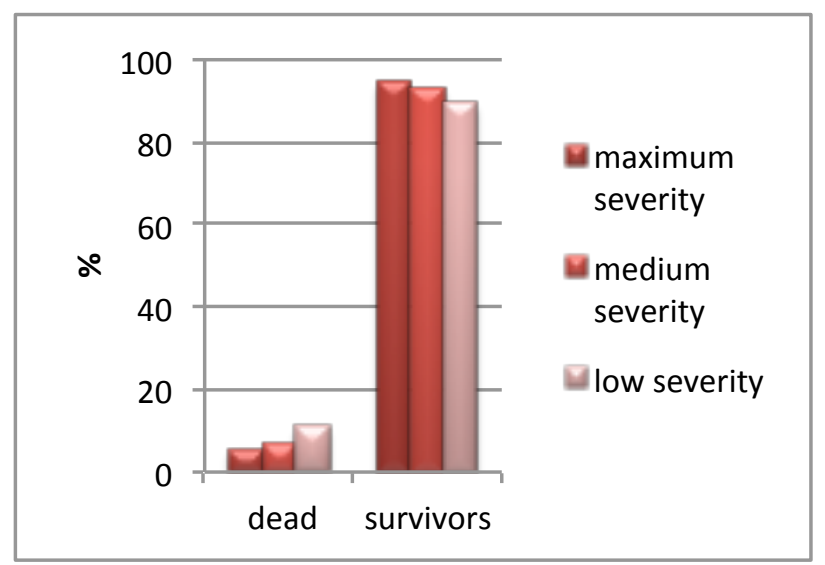

Fig. 2. Percentage of dead and survivors for each event severity level (maximum, medium, low).

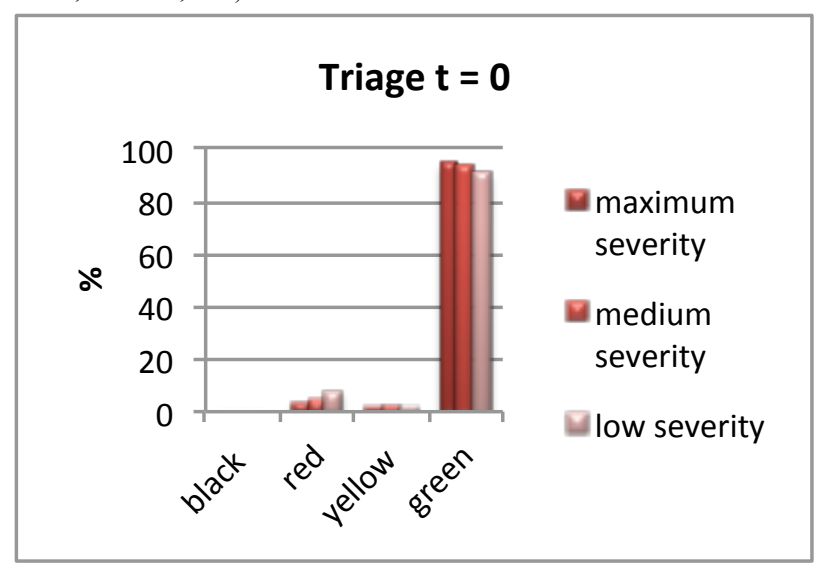

Fig. 3. Percentage of color code triage (black, red, yellow, green) for each event severity level (maximum, medium, low) at initial time of event $(\mathrm{t}=0)$.

\section{B. Simulation 2}

Simulation 2 shows the physiological patient evolution after intervention with different Therapeutic Maneuvers:

2.a therapy code $=1$ (i.e., O2);

$2 . b$ therapy code $=3$ (i.e., decontamination).

The simulated scenario has the following features:

- $\quad$ event code = IHS;

- $\quad$ number of people in area $=1000$;

- $\quad$ event radius $=100[\mathrm{~m}]$;

- $\quad$ event severity = 1 (maximum);

- $\quad$ severity probability distribution = gaussian;

- time horizon $\mathrm{T}=100[\mathrm{~h}]$;

- $\quad$ intervention time $=2[\mathrm{~h}]$.

Simulation 2.a shows in Fig. 4 the evolution of $x(t)$ and $v(t)$ for each variable in the set (ABCDE) for $25^{\circ}, 50^{\circ}$ and $75^{\circ}$ percentile untreated patient (on the left-hand side) and treated $\left(\mathrm{O}_{2}\right)$ patient (on the right-hand side). In particular, Fig. 5 considers only the median patient $\left(50^{\circ}\right.$ percentile $)$ and shows the evolution of $\mathrm{x}(\mathrm{t})$ and $\mathrm{v}(\mathrm{t})$ for each variable in the set (ABCDE). In the right-hand side of Fig. 5, it is shown the evolution with $\mathrm{O} 2$ therapy: with this therapeutic maneuver, there is an improvement for $\mathrm{A} 1, \mathrm{~B} 1, \mathrm{~B} 2, \mathrm{~B} 3, \mathrm{C} 1, \mathrm{C} 2$, and D1 variables, but not for example for E1 (Fig. 6), where the variable E1 maintains the same evolution between untreated (Fig. 6, left-hand side) and treated (Fig. 6, right-hand side) patients.

Simulation 2.b shows in Fig. 7 the evolution of $x(t)$ and $v(t)$ for each variable in the set (ABCDE) for $25^{\circ}, 50^{\circ}$ and $75^{\circ}$ percentile untreated patient (on the left-hand side) and treated (decontamination) patient (on the right-hand side). In detail, Fig. 8 considers only the median patient and shows the evolution of $\mathrm{x}(\mathrm{t})$ and $\mathrm{v}(\mathrm{t})$ for each variable in the set (ABCDE). In the right-hand side of Fig. 8, it is shown the evolution in presence of decontamination therapy: with this therapeutic maneuver there is an improvement for each physiological variable. 


\section{Simulation 2.a:}

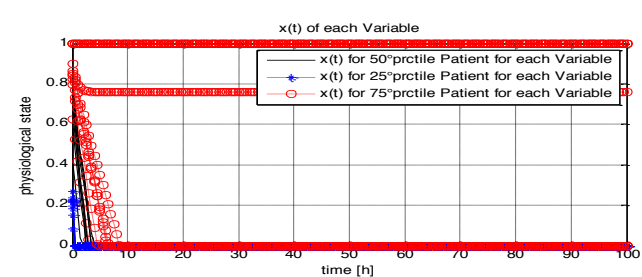

time $[\mathrm{h}]$

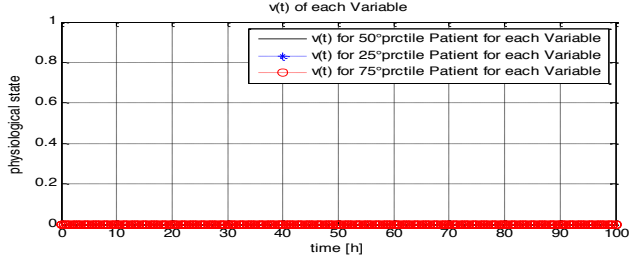

TREATED $x$ (t) of each Variable

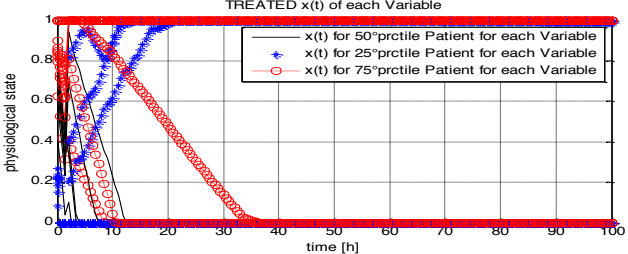

time $[\mathrm{h}]$

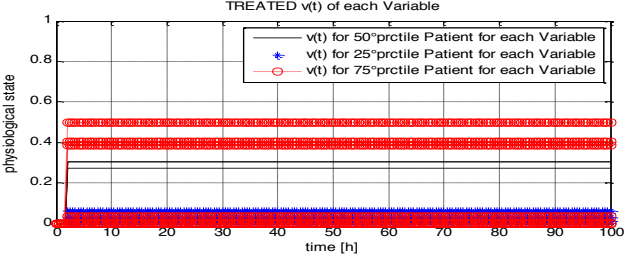

Fig. 4. On the left-hand side, it is shown the evolution of $25^{\circ}, 50^{\circ}$ and $75^{\circ}$ percentile UNTREATED patient $(x(t)$ and $v(t))$ for each variable in the set $(A B C D E)$; on the right-hand side, it is shown the evolution of $25^{\circ}, 50^{\circ}$ and $75^{\circ}$ percentile TREATED $\left(0_{2}\right)$ patient $(x(t)$ and $v(t))$ for each variable in the set $(A B C D E)$.
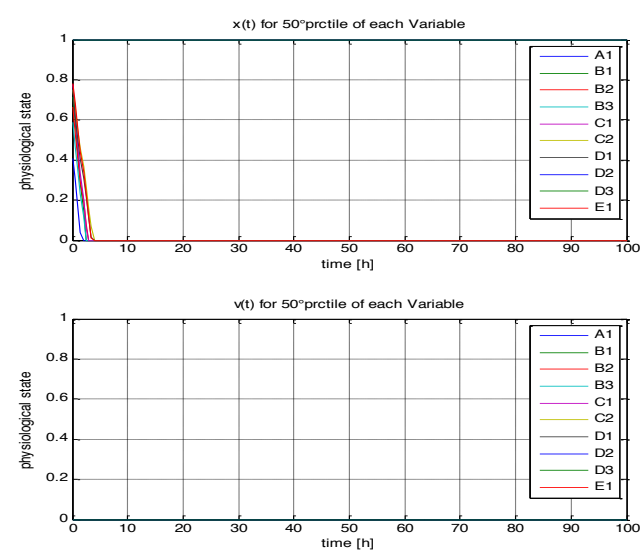
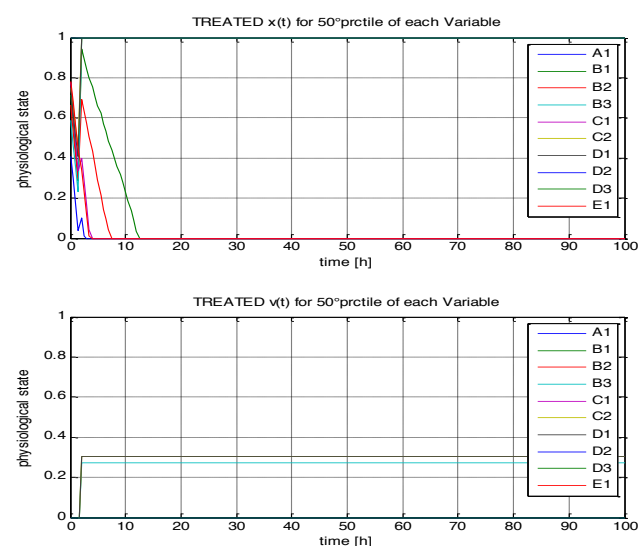

Fig. 5. On the left-hand side, it is shown the evolution of the median UNTREATED patient $(x(t)$ and $v(t))$ for each variable in the set $(A B C D E)$; on the right-hand side, it is shown the evolution of the median TREATED $\left(0_{2}\right)$ patient $(x(t)$ and $v(t))$ for each variable in the set $(A B C D E)$.

Behavior of variable E1 (Extra damage/Exposure):

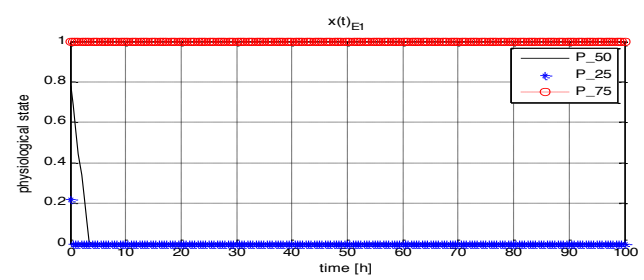

$\left.v^{(t)}\right)_{E 1}$

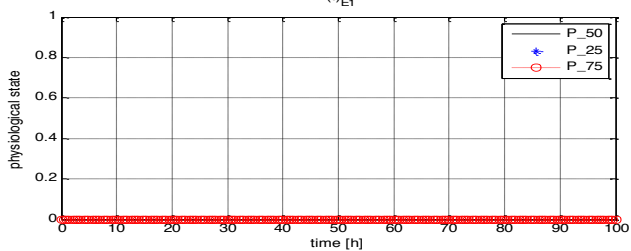

TREATED $\times\left(t_{E} 1_{1}\right.$

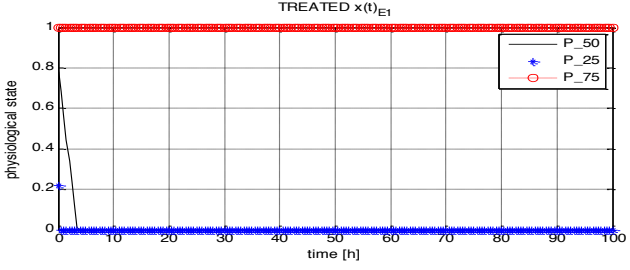

TREATED $v^{(t)} E_{E 1}$

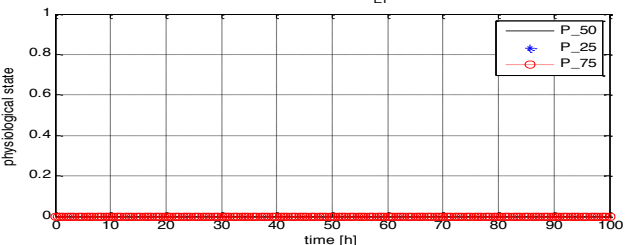

Fig. 6. On the left-hand side, it is shown the evolution of $25^{\circ}, 50^{\circ}$ and $75^{\circ}$ percentile UNTREATED patient $(\mathrm{x}(\mathrm{t})$ and $\mathrm{v}(\mathrm{t}))$ for variable E1; on the right-hand side, it is shown the evolution of $25^{\circ}, 50^{\circ}$ and $75^{\circ}$ percentile TREATED $\left(0_{2}\right)$ patient $(x(t)$ and $v(t))$ for variable E1. 
Simulation 2.b:

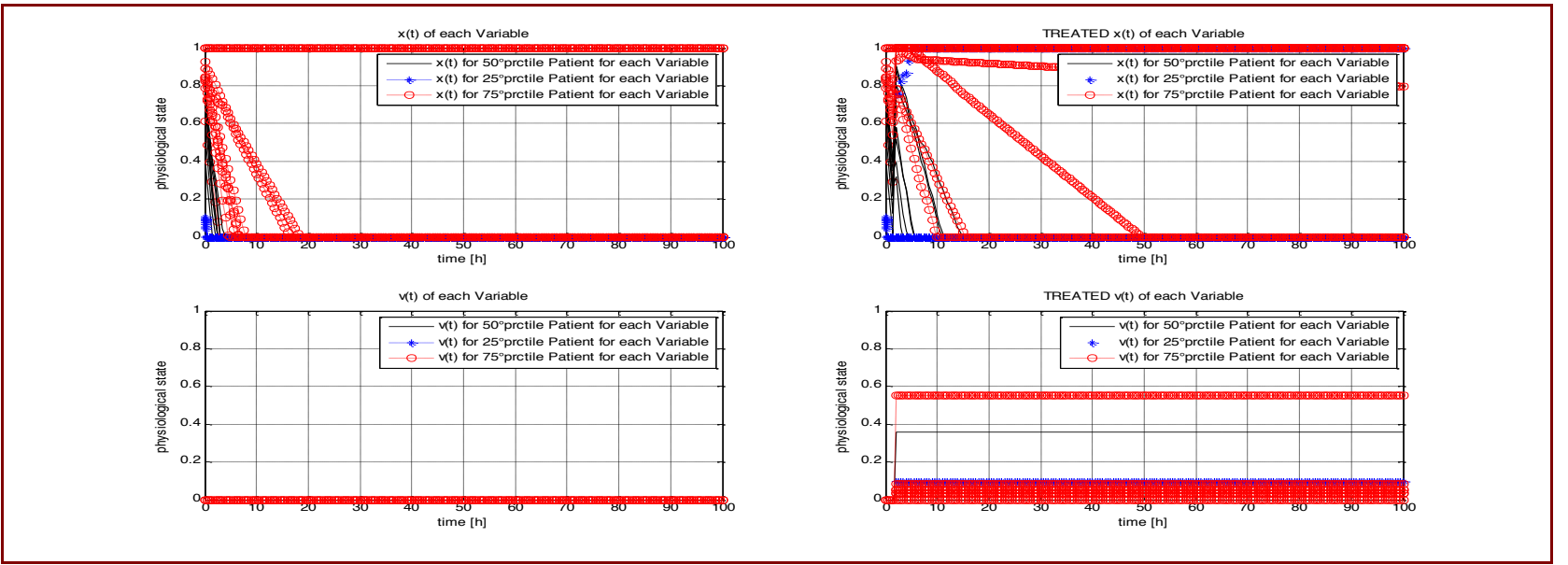

Fig. 7. On the left-hand side, it is shown the evolution of $25^{\circ}, 50^{\circ}$ and $75^{\circ}$ percentile UNTREATED patient $(x(t)$ and $v(t))$ for each variable in the set $(\mathrm{ABCDE})$; on the right-hand side, it is shown the evolution of $25^{\circ}, 50^{\circ}$ and $75^{\circ}$ percentile TREATED (decontamination) patient $(x(t)$ and $v(t))$ for each variable in the set (ABCDE).

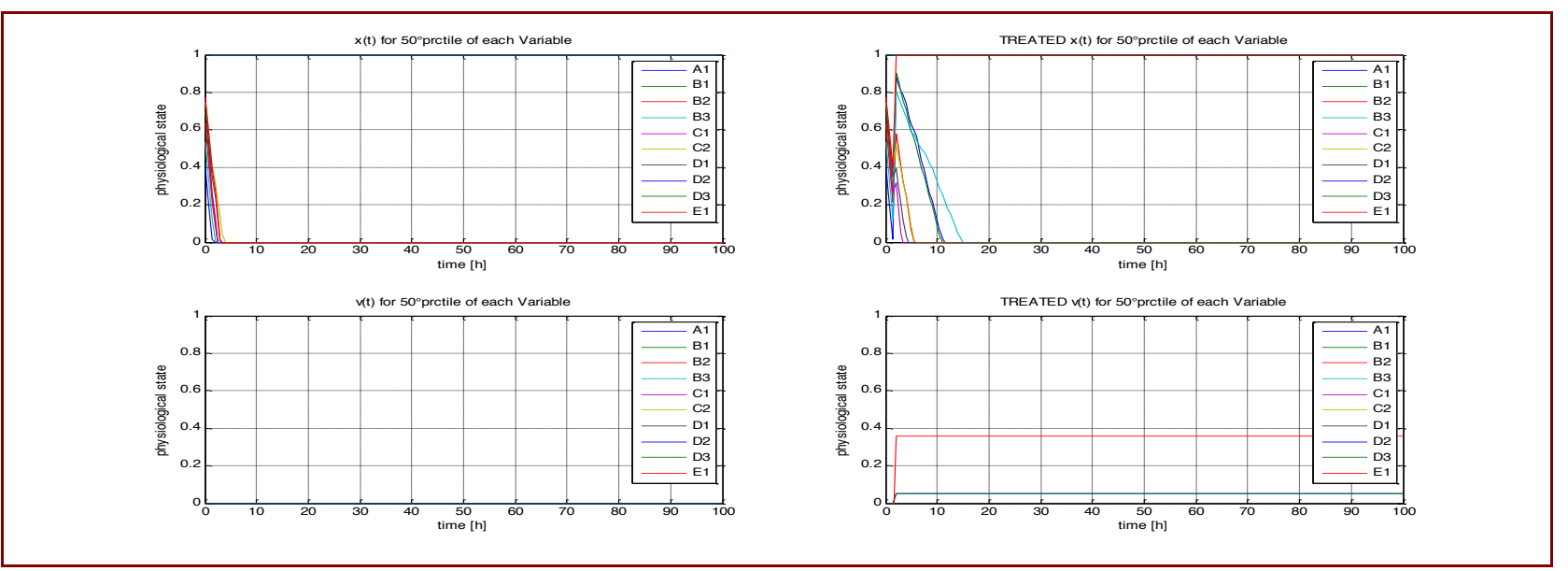

Fig. 8. On the left-hand side, it is shown the evolution of median UNTREATED patient $(x(t)$ and $v(t))$ for each variable in the set $(A B C D E)$; on the right-hand side, it is shown the evolution of median TREATED (decontamination) patient $(x(t)$ and $v(t))$ for each variable in the set $(A B C D E)$.

\section{CONCLUSIONS}

We have developed and implemented a mathematical model of the physiological patient evolution during/after physical and toxic trauma events. The evolution of the value of 10 physiological variables (i.e., A1, B1, B2, B3, C1, C2, D1, $\mathrm{D} 2, \mathrm{D} 3$, and E1) is simulated in different physical or toxic trauma incident scenarios; the ultimate goal is to predict adverse outcomes with simplified methods for triage and personalize the treatment of the patients with available therapeutic maneuvers.

The results could provide a benchmark for potential introduction and proliferation of applications to be employed in real operation during MIs medical response, with improvement of safety and security of citizens. In particular, it will be possible the development of health monitoring applications or web services, aiming to: saving data remotely; producing reports on the health status of each patient; supporting decision-making during MIs, where medical staff acts in limited time, under pressure, without a second chance, outside their own medical specialties and with high load of casualties.

The future prospective is to link this physiological patientevolution model to a logistic model in order to: handle/request stockpiles and available resources during emergency; plan them in the preparedness phase for particular events, as mass gatherings; analyze old and new vulnerabilities (e.g., the overpopulation and how this effects healthcare) to enhance the resilience capacity and the better integration of healthcare systems. These models will be implemented in telemedicine tools to insure an interoperability standardization for medical response during MIs. Such tools could be used also during interactive training by emergency medical practitioners (which cannot be trained in real situations as MI) in order to "learn by doing". 


\section{ACKNOWLEDGMENT}

The research leading to these results has been partially supported by the EU-funded research projects EDEN, PULSE, IMPRESS under the European Union Seventh Framework Programme for Research [FP7/2007-2013].

\section{REFERENCES}

[1] Lennquist S., et al. (2012). Medical Response to Major Incidents and Disasters. Springer.

[2] Klyman, Y., Kouppari, N., Mukhier, M. (2007). World Disaster Report 2007. International Federation of Red Cross and Red Crescent Societies, Geneva.

[3] Walter, F.G, Klein, R., Thomas, R.G., et al. (2003). Advanced Hazmat Life Support $^{\mathrm{TM}}$ (AHLS), Provider Manual, Third Edition, University of Arizona, Emergency Medicine Research Center, American Academy of Clinical Toxicology. (pp. 4-19; 51-65; 186-194).

[4] Alywin, C.J. (2006). Reduction in mortality in urban mass casualty incidents - analysis of triage, surgery and resources use after the London bombings on July 7, 2005. Lancet 368:2219 - 2225 .

[5] Smith, E., Waisak, J., Archer, F. (2009). Three decades of disasters - a review of Disaster - specific literature from 1977 - 2009. Prehosp Disaster Med 24:306 - 311 .

[6] Frykberg, E.R. (2002). Medical Management of disaster and mass casualties from terrorist bombings - how can we cope? J Trauma 53:201 -212 .

[7] Lennquist, S. (2003). The importance of maintaining simplicity in planning and preparation for major incidents and disaster. Int J Disaster Med 2004:5 - 9

[8] Lennquist, S. (2003). Promotion of disaster medicine to a scientific discipline - a slow and painful but necessary process. Int J Disaster Med 2:95- 99 .

[9] Walter F., Dedolph R., Kallsen G., et al. (1992). Hazardous materials incidents: A one-year retrospective review in Central California. Prehospital and Disaster Medicine 7:151-156.
[10] Bertazzi, PA. (1989) Industrial disasters and epidemiology - A review of recent experiences. Scand J Work Environ Health 15:85-100.

[11] Kales, S.N., Castro, M.J., Christiani, D.C. (1996). Epidemiology of hazardous materials responses by Massachusetts district HAZMAT teams. J Occup Environ Med 38:394-400.

[12] Nutbeam, T., Boylan, M. (2013). ABC of Prehospital Emergency Medicine. Wiley Blackwell.

[13] Moreira, L.B., Kasetsuwan, N., Sanchez, D., Shah, S., LaBree, L., McDonnell, P.J. (1999). Toxicity of topical anesthetic agents to human keratocytes in vivo. Journal of Cataract \& Refractive Surgery 25:975-80.

[14] Binder, S., Bonzo, S. (1989). Acute hazardous materials release. Am J public Health 79:1681.

[15] Burgess, J.L., Pappas, G.P., Robertson, W.O. (1997). Hazardous materials incidents: the Washington Poison Center experience and approach to exposure assessment. J Occup Environ Med 39:760-6.

[16] Ellis, D., Hooper, M. (2010). Cases in Pre-Hospital and Retrieval Medicine, 1st edition. Churchill Livingstone, Elsevier. Australia.

[17] Greaves, I., Porter, K. (2007). Pre-Hospital Care. Oxford University Press.

[18] Wyatt, J.P., Illingworth, R.N., Graham, C.A., Hogg, K. (2012). Emergency Medicine. $4^{\text {th }}$ ed. Oxford University Press.

[19] Waldmann, C., Soni, N., Rhodes, A. (2008). Critical Care. Oxford University Press.

[20] Singer, M., Webb, A.R. (2009). Critical Care. $3^{\text {rd }}$ ed. Oxford University Press.

[21] Cone, D.C., Koenig, K.L. (2005). Mass casualty triage in the chemical, biological, radiological, or nuclear environment. Eur J Emerg Med 12:287-302.

[22] Jenkins, J.L., McCarthy, M.L., Sauer, L.M., Green, G.B., Stuart, S., Thomas, T.L., Hsu, E.B. (2008). Mass-casualty triage: Time for an evidence-based approach. Prehospital Disast Med 23(1):3-8.

[23] Partridge, R.A., Proano, L., Marcozzi, D., et al. (2012). Disaster medicine. Oxford University Press. 